\title{
Practice of contemporary dance improves cognitive flexibility in aging
}

\author{
Olivier A. Coubard ${ }^{1}$, Stéphanie Duretz ${ }^{1}$, Virginie Lefebvre ${ }^{1}$, Pauline Lapalus ${ }^{2}$ and Lena Ferrufino ${ }^{1,3}$ \\ ${ }^{1}$ The Neuropsychological Laboratory, CNS-Fed, Paris, France \\ ${ }^{2}$ Centre Mémoire de Ressources et de Recherche Paris Nord, Groupe Hospitalier Lariboisière-Fernand Widal, Paris, France \\ ${ }^{3}$ Groupe de Recherche Apprentissage et Contexte, Ecole des Hautes Etudes en Sciences Sociales, Paris, France
}

\section{Edited by:}

Rudy Castellani, University of

Maryland, Baltimore, USA

Reviewed by:

Luis Francisco Gonzalez-Cuyar, University of Maryland School of Medicine, USA

Fabio Tavora, University of Maryland

School of Medicine, USA

Jonathon Edward Heath, University of

Maryland Medical Center, USA

*Correspondence:

Olivier A. Coubard, The

Neuropsychological Laboratory,

CNS-Fed, 39 rue Meaux 75019 Paris,

France.

e-mail: olivier.coubard@cns-fed.com
As society ages and frequency of dementia increases exponentially, counteracting cognitive aging decline is a challenging issue for countries of the developed world. Previous studies have suggested that physical fitness based on cardiovascular and strength training helps to improve attentional control in normal aging. However, how motor activity based on motor-skill learning can also benefit attentional control with age has been hitherto a neglected issue. This study examined the impact of contemporary dance (CD) improvisation on attentional control of older adults, as compared to two other motor training programs, fall prevention and Tai Chi Chuan. Participants performed setting, suppressing, and switching attention tasks before and after 5.7-month training in either CD or fall prevention or Tai Chi Chuan. Results indicated that $C D$ improved switching but not setting or suppressing attention. In contrast, neither fall prevention nor Tai Chi Chuan showed any effect. We suggest that $C D$ improvisation works as a training for change, inducing plasticity in flexible attention.

\section{Keywords: aging, attentional control, motor activity, contemporary dance, improvisation, plasticity}

\section{INTRODUCTION}

In recent years, there has been an increasing interest in the aging of cognitive processes and of their neural underlying mechanisms. While some studies have pointed out cognitive improvements with age (Tentori et al., 2001), most of them have reported a decline in functions such as perception (Blake et al., 2008), memory (Lister and Barnes, 2009), or motor behavior (Hsiao-Wecksler, 2008). Nevertheless, neural networks and cognitive processes do not evolve uniformly in normal aging. Consistent with a precocious decline of prefrontal areas of the brain (Rajah and D'Esposito, 2005; Raz and Rodrigue, 2006), executive functions show earlier and larger decline than other functions in older adults (Bherer et al., 2004). Executive functions, the mental capacities for formulating goals, planning how to achieve them, and carrying out the plans effectively, include a wide variety of functions such as volition, planning, purposive action, and effective performance (Lezak, 1995). To avoid confusion, we use the terms "attentional control" to restrict executive functions to their attentional dimension (Coubard et al., 2011). Attentional control is the ability to maintain goal-directedness by sustaining information-processing activity over time in the face of distraction, temporarily stopping the activity to respond to other information, and coordinating the course of concurrent activities (Parasuraman, 2000). Stuss and colleagues have described seven tasks of attentional control (concentrating, setting, preparing, sustaining, suppressing, switching, and sharing), which involve six cognitive processes (energizing, inhibiting, adjusting, monitoring, controlling, and task setting), for which the prefrontal correlate has been identified in at least three of them: superior medial for energizing, left lateral for task setting, and right lateral for monitoring (Stuss et al., 1995; Stuss and Alexander, 2007). In normal aging, a deficit has been reported for almost all tasks of the prefrontal attentional system: setting (Bugg et al., 2006; Zook et al., 2006), preparing (Bherer and Belleville, 2004; Vallesi et al., 2009; Coubard et al., 2011), sustaining (Rypma et al., 2007), suppressing (Verhaeghen and Cerella, 2002; Amieva et al., 2004; Coubard et al., 2011), switching (Ashendorf et al., 2008; Sorel and Pennequin, 2008; Coubard et al., 2011), and sharing (Verhaeghen and Cerella, 2002; Chaparro et al., 2005). Given the importance of attentional control in all cognitive functions, these deficits are likely to have deleterious impact in daily activities of older adults (Vaughan and Giovanello, 2010). In this context, it is urgent to develop strategies to prevent attentional control decline and preserve independent living for a successful aging (Hank, 2011).

Several cognitive and physical interventions have been designed to target attentional control in older adults with beneficial effects. Using a dual-task paradigm, Bherer et al. $(2005,2006,2008)$ showed that cognitive training could substantially improve timesharing skills of older adults. Nevertheless, older adults as compared to young ones are less likely to bypass the central bottleneck, i.e., to automatize (Maquestiaux et al., 2008, 2010), consistent with higher training-induced recruitment of the dorsolateral prefrontal cortex (DLPFC; Erickson et al., 2007; Mozolic et al., 2010). Since the pioneer research by Spirduso (1975), physical interventions have also been found to benefit cognition. Specifically, several studies have shown that physical fitness, based on cardiovascular and/or strength conditioning, can have indirect beneficial impact on cognition, particularly attentional control (for reviews, see Kramer et al., 2005; Kramer et al., 2006; Schäfer et al., 2006; Kramer and Erickson, 2007; Hillman et al., 2008). In 
these studies, the rationale is the following: cognitive decline may be partially caused through cerebrovascular insufficiency, which tends to increase with age; thus cardiovascular conditioning, which enhances cerebrovascular sufficiency by increasing aerobic capacity and/or cardiac output through increased stroke volume and oxygen extraction, may also be able to reduce aged-related decline due to cerebrovascular insufficiency (Hawkins et al., 1992). Comparing older adults (63-82 years) assigned to either a 10-week aquatic exercise program or a control group without physical activity, Hawkins et al. (1992) used a dual-task paradigm and showed higher alternation speed and time-sharing efficiency after training in the exercise group as compared to the control group. Kramer et al. (1999) trained 124 older adults (60-75 years) to either aerobic (walking) or anaerobic (stretching and toning) exercise for 6 months. After training, the aerobic group performed better in three tests measuring task switching, response compatibility, and stopping, as compared to the anaerobic group. Importantly after training, the aerobic group had higher rate of oxygen consumption than the anaerobic group and both groups were equivalent in non-switch trials, response time in compatible trials, and simple reaction time in stopping, suggesting that cardiovascular fitness specifically improved attentional control (Kramer et al., 1999). More recently, Renaud et al. (2010) showed that older adults (67 years in average) trained to 12 -week aerobic training (stretching, fast walking, and aerobic dancing) improved their cardiorespiratory capacity together with their preparing attention ability in a choice reaction time task, as compared to a control group without any physical activity. There are several arguments in animal studies to explain how physical fitness vs. motor activity may improve cognition by enhancing neural plasticity through neurogenesis, synaptogenesis, or angiogenesis (e.g., Black et al., 1990; Neeper et al., 1995; Churchill et al., 2002). Using functional magnetic resonance imaging (fMRI) in humans, Colcombe et al. (2004) invited aerobic vs. anaerobic-trained older adults to perform a flancker paradigm, involving attentional control, before and after a 6-month training. In the aerobic group, there was greater taskrelated activity in prefrontal and parietal cortices and lower activity in the anterior cingulate cortex (ACC), known to monitor conflict in the attentional system.

How motor activity, particularly dance, can be beneficial for cognition is however a neglected question. Numerous qualitative reports have suggested that dance can benefit physical and/or cognitive health in aging (e.g., Houston, 2005; Libster, 2006; Lima and Vieira, 2007; Stacey and Stickley, 2008; Kattenstroth et al., 2009). To our knowledge, there are few scientific studies examining influence of dance on cognitive functions in older adults. In a cross-sectional study, Verghese (2006) found no difference in cognitive performance, including attentional control as assessed by verbal fluency and the Trail making test-B, between 24 older social dancers (aged 80 years in average with 36.5 years of dance experience, 4.3 days a month) and 84 age-matched non-dancers. In an intervention study, Alpert et al. (2009) trained 13 women aged 52-88 years to jazz dance for 15 weeks - there was no control group. Three assessments at weeks 1-2, 8-9, and after training showed no cognitive improvement as assessed by the Mini-Mental State Examination (MMSE). Eyigor et al. (2009) assigned 37 older women over 70 years to either an 8 -week Turkish folkloric dance program or to a control group without activity, but cognitive performance was not assessed. Kattenstroth et al. (2010) examined the impact of 16.5 years of ballroom dancing ( $1.33 \mathrm{~h}$ a week) on sensori-motor and cognitive functions. Twenty-four amateur dancers aged 61-94 years were compared to 38 age-matched nondancers in lifestyle, cognitive performance, reaction time, balance, motor performance, and tactile performance. The dance group performed better than the control group in almost all tests, and individual analysis revealed that the dance group lacked individuals with poor performance. Studies on techniques other than dance such as Hatha yoga have reported non-improvements of cognitive function, including attention control, after several months of practice (e.g., Oken et al., 2006).

Taken together, these studies point out the importance of dissociating between physical fitness training based on cardiovascular and/or strength on the one hand, and motor activity training focusing on motor skills on the other. In the field of dance, there is also a need to differentiate between the different types of danceballet, modern, contemporary, folkloric, social, dance/movement therapy, etc. Finally, studies targeting the influence of dance on attentional control are lacking contrasting with the literature on physical fitness impact in cognitive aging.

The goal of our study was to examine the influence of contemporary dance (CD) on attentional control in normal aging, as compared to other motor interventions. The $\mathrm{CD}$ training received by the participants was based on specific theoretical, technical, and education principles with a focus on improvisation (FerrufinoVargas, 2010). In two control groups, older participants were trained to either fall prevention or Tai Chi Chuan. Three components of attentional control were assessed. First, setting attention, which requires establishing a sequence of appropriate processes to complete an up-coming task (Stuss et al., 1995), was assessed using arithmetic word problems (Luria and Tsvetkova, 1967). Second, suppressing attention, which is required when automatic responses need to be inhibited (Stuss et al., 1995), was appreciated using the Stroop test (Stroop, 1935). Third, switching attention, which demands shifting attention between tasks requiring different stimulus-response pairings (Stuss et al., 1995), was assessed using the Rule shift cards sorting test (Wilson et al., 1998). We hypothesized that CD improvisation might enhance attentional control contrary to the two other training programs based on stereotyped behaviors. As our training programs targeted motor activity and not physical fitness, we neither expected nor measured changes in physical fitness.

\section{MATERIALS AND METHODS PARTICIPANTS}

One hundred ten French natives gave their consent to participate in the study, which adhered to the tenets of the Declaration of Helsinki and was approved by the local ethics committee (Ecole des Hautes Etudes en Sciences Sociales, Paris). They were righthanded, had no known neurological or psychiatric disorders, and had normal or corrected-to-normal vision. All were unaware of the goal of the experiment. Table 1 details the participants' sex, age, years of education (Lezak, 1995), socio-cultural level defined as the years of education weighted by socio-professional experience according to Poitrenaud's recommendations (Kalafat et al., 
Table 1 | Number (Gender) or Mean \pm SD (Age, Education, Socio-cultural level, MMSE, code) for the groups of participants (CD: contemporary dance; fall: fall prevention; Tai: Tai Chi Chuan).

\begin{tabular}{lccc}
\hline & CD & Fall & Tai \\
\hline Sex (women/men) & $16 / 0$ & $64 / 3$ & $24 / 3$ \\
Age (years) & $73.6 \pm 5.4$ & $74.7 \pm 6.7$ & $71.5 \pm 7.4$ \\
Education (years) & $9.9 \pm 4.4$ & $9.2 \pm 3.9$ & $11.0 \pm 3.6$ \\
Socio-cultural level (/4) & $3.1 \pm 0.9$ & $2.9 \pm 0.9$ & $3.3 \pm 0.8$ \\
MMSE (/30) & $27.6 \pm 1.5$ & $27.0 \pm 2.3$ & $27.6 \pm 2.0$ \\
Code (/20) & $11.5 \pm 3.7$ & $12.1 \pm 2.6$ & $11.5 \pm 2.2$ \\
\hline
\end{tabular}

2003), and their score in the MMSE for general cognition (Folstein et al., 1975), and in the WAIS-R code for processing speed (Wechsler, 1989).

Sixteen participants aged 64.9-83.0 years made up the experimental or CD group. A first control group was made up of 67 participants aged 59.9-89.2 years trained in fall prevention, a second one was made up of 27 participants aged 59.5-88.7 trained in Tai Chi Chuan.

The three groups were matched in sex $\left(\chi^{2}<1\right.$ in $2 \times 2$ comparisons), age (one-way ANOVA, $F_{2,107}=2.25, P>0.05$ ), years of education $\left(F_{2,107}=2.04, P>0.05\right)$, socio-cultural level $\left(F_{2,107}=2.92, P>0.05\right)$, in their score in the MMSE $(F<1)$ and in the Code $(F<1)$.

The three groups were matched in past physical activity as measured by the years of practice in each of the following activities. Sixty of them had done $2.0 \pm 1.8$ years of fall prevention in the past, 33 of them had done $4.5 \pm 3.8$ years of gymnastics, 23 of them had done $3.4 \pm 3.1$ years of aquagymnastics, and 13 of them had done $1.3 \pm 0.6$ years of Tai Chi Chuan. The difference between the three groups did not reach statistical significance for each activity (Kruskal-Wallis, $P>0.05$ ).

\section{MATERIAL, PROCEDURE, AND MEASURES Neuropsychological assessment}

All groups underwent three pencil-and-paper tests (arithmetic word problems, Stroop test, and Rule shift cards test) before and after the training intervention. The order of tests was counterbalanced as closely as possible in a Latin square design.

Arithmetic word problems. Based on pioneering research by Luria and Tsvetkova (1967) on arithmetic word problems with increasing planning demand from levels 1 to 8, Aubin et al. (1994) have shown that the breakdown for patients with frontal lobe lesion was between levels 3 and 4 . Indeed, patients managed almost all problems of levels 1-3 and failed those of levels 4-8. Material. We used two arithmetic word problems, one of level 3 and the other of level 4. The low planning demand problem (level $3)$ required solving a two-step problem such as $a+(a+b)=x$ or $a+(a-b)=x$. The high planning demand problem (level 4 ) required solving a multiple-step problem such as $a+n=x$ then $x+m=z$ or $a+(a+b)+(a+b)-c=x$. The terms of the problems were different between pre- and post-test, but their structure and difficulty were equivalent. Procedure. Participants had to solve low then high planning demand problems, in writing, as accurately and fast as possible. Measures. For each problem, we measured accuracy ( 0 or 1$)$ and time for correct responses in seconds.

Stroop test. Material. Three sheets corresponding to three conditions each contained 10 practice and 100 test stimuli. Stimuli of condition 1 were red, green, or blue crosses. The stimuli of condition 2 were the color names "red," "green," or "blue," printed in black ink. The stimuli of condition 3 were the color names printed in incongruent red, green, or blue ink. This material conformed to GREFEX standards (Meulemans, 2008), except that crosses replaced rectangles in condition 1 to fit condition 3 better. Procedure. In condition 1 (naming task), participants had to name the crosses' color. In condition 2 (reading task), they had to read the words. In condition 3 (interference-naming task), they had to name the color words while preventing word reading. All conditions were stopped at a maximum of $90 \mathrm{~s}$ to prevent fatigue effects. Measures. For each condition, we measured the time $(90 \mathrm{~s}$ or shorter), the number of correct responses and of errors. For time shorter than $90 \mathrm{~s}$, scores were extrapolated to 90 (e.g., 105 responses in $75 \mathrm{~s}$ led to $90 / 75 \times 105=126$ responses). We first calculated an interference ratio ranging 0 (null) to 1 (maximum) as the number of correct responses in the condition 3 , divided by that in condition 1 . The interference error rate was the difference in percentage of errors between conditions 3 and 1 .

Rule shift cards test. This test was taken from the Behavioral Assessment of the Dysexecutive Syndrome battery (Wilson et al., 1998). Material. The stimuli were 21 spiral-bound $10.5 \times 14.8-\mathrm{cm}$ playing cards numbered 0-20; they were either red or black. Procedure. Participants completed two conditions. For each condition, the instruction was displayed next to the stimuli and explained by the examiner. In condition 1 , they had to respond "Yes" to a red card and "No" to a black card. Card $n 0$ was devoted to practice. In condition 2, participants had to respond "Yes" if the card was the same color as the previously displayed card, and "No" if it was a different color. Card $n 0$ allowed the participant to respond to card $n 1$. In all trials, each card remained on the table until participants had responded orally, and the rule was visible next to the card throughout the test. Measures. We first calculated the profile score out of 4 based on errors and execution time in condition 2: $0,1-3,4-6,7-9$, or more than 9 errors led to a score of 4,3 , 2,1 , or 0 , respectively, which was decremented by 1 if time was over $67 \mathrm{~s}$ (Wilson et al., 1998). We also measured the switch error rate as the percentage of failure in switching items of condition 2. Indeed, this condition contains 11 non-switching items and 9 switching items. In the former, responding according to the rule of condition 1 ("Yes" for red, and "No" for black) or that of condition 2 ("Yes" for a color shift, and "No" for no color shift) provided the same answer, so that a putative switch error was invisible to the examiner. In contrast, in the latter (cards $n 3-5,10,13,15-17$, and 19), responding following the first rule in condition 2 resulted in a switch error, as the answer differed from that of condition 1 .

\section{Training programs}

Participants were trained to either CD or fall prevention or and Tai Chi Chuan for $5.9 \pm 1.2,5.7 \pm 1.1$, and $5.6 \pm 0.4$ months, respectively $(F<1)$. Participants did not have a choice in the training in 
which they were recruited, which was determined by the district where they lived in the Paris region. The training was conducted by professional instructors and supervised by a senior teacher, each in their specialty (CD, fall prevention, or Tai Chi Chuan). The frequency of all training programs was once a week, and each session lasted $1 \mathrm{~h}$. In all training programs, music could be used during up to $50 \%$ of session duration. Participants did not take part in other motor training programs during the intervention period. Importantly, the three training programs focused on movement: motor creativity in CD, motor skills to avoid falls in fall prevention, motor sequences developing internal energy in Tai Chi Chuan. The three training programs only varied in their degree of exploration of the movement: improvisation in CD vs. reproduction and/or modulation in fall prevention and Tai Chi Chuan (Ferrufino-Vargas, 2010). None of the programs developed cardiovascular condition and/or strength.

Contemporary dance. A CD session focused on improvisation inviting participants to produce rather than reproduce or repeat movements. (1) Opening was adapted to the characteristics and needs of the group (e.g., variations of the action of walking, free dance on a popular music). (2) Warm-up and preparation to dance. Body was awakened by passive and active movements of joints, and movements of muscular stretching promoting coordination, link between breath and movement, and body positioning and alignment. (3) Improvisation. A theme was proposed by the teacher, it could be a word, an action, an idea, an object, some music, or a location. Based on this constraint, improvisation was organized around four steps: (i) individual exploration of the theme; (ii) exploration in pair or more taking into account the presence of the others. The trainer proposed dance tools at steps (i) and (ii) to promote exploration and the use of resources of each individual and of the exercise. In a step (iii), each group presented the work developed in (i) and (ii). In a step (iv), participants were invited to improvise in a solo, developing a natural movement for expressing their own sensations. (4) Closure. Participants cooled down through breath and massages. For a 1-h session, sections of $5,20,30$, and 5 min were dedicated to the stages (1), (2), (3), and (4), respectively.

Fall prevention. Fall prevention training emphasized the development of balance and of lower limbs. A session was organized as follows. (1) Warm-up and stretching. (2) Then, participants stimulated the visual, vestibular, kinesthesic, and proprioceptive functions in a specific way to optimize each of them. (3) Workshops were organized using objects: as example, participants stepped over obstacles, walked on foam rubbers or on small bags of sand, walked on a rope, etc. The training focused on motor skills of muscular lower-limb groups ensuring postural stability, as well as accuracy and amplitude of movements. (4) Cooling down and stretching. The training was performed individually and in pairs. For a 1-h session, 10, 20, 20, and $10 \mathrm{~min}$ were dedicated to stages (1) to (4).

Tai Chi Chuan. Traditional Chinese martial art, Tai Chi Chuan emphasizes the development of internal energy and of dynamic force (jing), and develops balance, calm, and concentrating attention of fighters. Tai Chi Chuan movements and sequences of movements were taken from different schools (basic, Pa-Kua, Wu, Yang, etc.). First, participants worked on relaxation (song) allowing the steady flow of movements and of coordination. Once relaxed, participants developed internal force (peng jing) making the link between the different parts of the body while maintaining relaxation. In other words, when a body part moved, the entire body moved, when a body part stopped, the whole body stopped. In this stage, movements were controlled through the practice of tangential and rotational forces, whose energy took root in the feet and below the navel (dantian), and were transferred all the way to the hands. The beginning of the training was performed slowly to feel the movements of vital energy (qi). Then participants could accelerate their movements and eventually practice movements of releasing energy (fa chin) with increasing amplitude. Tai Chi Chuan was practiced individually and in pairs for exercises requiring interaction such as hand thrusts.

\section{Data analysis}

All measures were submitted to analyses of variance (ANOVAs) with Group (three levels: CD vs. fall prevention vs. Tai Chi Chuan, or two levels: CD vs. fall prevention, and CD vs. Tai Chi Chuan) as between-participant factor, and Period (two levels: pre-test vs. post-test) as within-participant factor. Post hoc tests were calculated using Newman-Keuls (NK) method. We used statistica 7.0 (StatSoft, USA) for all analyses. Distributional information was given by SD for Participants and SE for Results.

\section{Additional analysis}

As differences in group sample sizes may have led to a statistical bias, analyses were re-run with random samples of the two control groups to adequate with the CD group sample size. New control groups were built by randomly selecting 20 participants in each of the fall prevention and Tai Chi Chuan groups. Table 2 details participants' sex, age, years of education, socio-cultural level, and their score to the MMSE and to the code. Twenty participants aged 60.8-89.2 years made up the new fall prevention group, and 20 others aged 59.6-88.7 years made up the new Tai Chi Chuan group.

The three groups were matched in gender $\left(\chi^{2}<1\right.$ in $2 \times 2$ comparisons), age (one-way ANOVA, $F<1$ ), years of education $(F<1)$, socio-cultural level $\left(F_{2,53}=1.43, P>0.05\right)$, in their score to the MMSE $(F<1)$, and to the Code $(F<1)$. The three groups were also matched in past physical activity as measured

Table 2 | Number (Gender) or Mean \pm SD (Age, Education, Socio-cultural level, MMSE, code) for the groups of participants (CD: contemporary dance; fall: fall prevention; Tai: Tai Chi Chuan) in the additional analysis.

\begin{tabular}{lccc}
\hline & CD & Fall & Tai \\
\hline Sex (women/men) & \multicolumn{1}{c}{$16 / 0$} & $19 / 1$ & $19 / 1$ \\
Age (years) & $73.6 \pm 5.4$ & $73.5 \pm 7.8$ & $71.8 \pm 6.9$ \\
Education (years) & $9.9 \pm 4.4$ & $10.1 \pm 3.7$ & $11.0 \pm 2.8$ \\
Socio-cultural level (/4) & $3.1 \pm 0.9$ & $3.0 \pm 0.9$ & $3.4 \pm 0.7$ \\
MMSE (/30) & $27.6 \pm 1.5$ & $28.1 \pm 1.4$ & $28.0 \pm 1.4$ \\
Code (/20) & $11.5 \pm 3.7$ & $12.7 \pm 2.1$ & $11.8 \pm 2.2$
\end{tabular}


by the years of practice in every activity. Twenty of them had done $2.0 \pm 1.8$ years of fall prevention in the past, 12 of them had done $3.6 \pm 3.9$ years of gymnastics, 11 of them had done $1.4 \pm 0.7$ years of Tai Chi Chuan, and 5 of them had done $5.2 \pm 4.1$ years of aquagymnastics. The difference between the three groups was non-significant for each activity (Kruskal-Wallis, $P>0.05)$.

Neuropsychological assessment, training programs, and data analysis were as in the main analysis.

\section{RESULTS}

\section{COUNTERBALANCING EFFECTS}

One-way ANOVA with Order as factor (three levels) did not show any statistical significant difference in pre-test period on accuracy and time $(F<1)$ of low and high planning problems, on the interference ratio and the interference error rate $(F<1)$ of the Stroop test, and on the profile score $\left(F_{2,107}=1.01, P>0.05\right)$ and the switch error rate $(F<1)$ of the Rule shift cards test; as well as in post-test period on accuracy and time $(F<1)$ of low planning problems, on accuracy $(F<1)$ and time $\left(F_{2,43}=1.22, P>0.05\right)$ of high planning problems, on the interference ratio $\left(F_{2,107}=1.32\right.$, $P>0.05)$ and the interference error rate $(F<1)$ of the Stroop test, and on the profile score $\left(F_{2,107}=2.11, P>0.05\right)$ and the switch error rate $\left(F_{2,107}=1.42, P>0.05\right)$ of the Rule shift cards test.

\section{NEUROPSYCHOLOGICAL ASSESSMENT}

Table 3 details the results for the three groups in the two periods. Consistent with the study goal, follow-up analyses compared CD to fall prevention on the one hand, and CD to Tai Chi Chuan on the other. Two-way ANOVAs with Group (two levels: CD vs. fall or CD vs. Tai) and Period (two levels: pre- vs. post-test) as factors did not show any statistically significant main effects $(P>0.05$, except a meaningless effect for time response of high planning arithmetic word problems when the number of trials was weak), thus the follow-up description was restricted to the interaction between Group and Period factors and related post hoc comparisons.

\section{Arithmetic word problems}

For accuracy of low planning problems, two-way ANOVAs with Group and Period as factors failed to show statistically significant interaction or significant post hoc test for CD compared to either fall prevention or Tai Chi Chuan (see Table 3). Response times were $5.20 \pm 6.97,9.04 \pm 3.25,5.45 \pm 4.70$, and $9.60 \pm 2.33$, $4.04 \pm 1.09,8.09 \pm 1.57 \mathrm{~s}$ for CD, fall prevention, and Tai Chi Chuan in pre- and post-test periods, respectively. Interaction and post hoc tests failed to reach statistical significance when comparing $\mathrm{CD}$ to either fall prevention $\left(F_{1,54}=1.06, P=0.308\right)$ or Tai Chi Chuan $(F<1)$.

In the high planning demand condition, there was neither significant interaction nor significant post hoc test for accuracy when comparing $\mathrm{CD}$ to either fall prevention or Tai Chi Chuan (see Table 3). Response times were 32.7 \pm 9.75, $31.3 \pm 5.41$, $24.0 \pm 13.8$, and $36.7 \pm 14.2,27.1 \pm 7.88,23.0 \pm 20.1 \mathrm{~s}$ for CD, fall prevention, and Tai Chi Chuan in pre- and post-test periods, respectively. Interaction and post hoc tests were not significant

Table 3 | Mean \pm SE of neuropsychological tests for the groups of participants (CD: contemporary dance, $N=16$; fall: fall prevention, $N=67 ;$ Tai: Tai Chi Chuan, $N=27$ ).

\begin{tabular}{|c|c|c|c|c|c|c|c|}
\hline & \multirow[t]{2}{*}{ CD } & \multirow[t]{2}{*}{ Fall } & \multirow[t]{2}{*}{ Tai } & \multicolumn{2}{|c|}{ CD vs. fall } & \multicolumn{2}{|c|}{ CD vs. tai } \\
\hline & & & & $\boldsymbol{F}$ & $\boldsymbol{P}$ & $\boldsymbol{F}$ & $\boldsymbol{P}$ \\
\hline Pre-test & $0.87 \pm 0.09$ & $0.82 \pm 0.04$ & $0.89 \pm 0.07$ & 1.10 & 0.296 & 1.72 & 0.197 \\
\hline Post-test & $0.69 \pm 0.10$ & $0.78 \pm 0.05$ & $0.88 \pm 0.08$ & & & & \\
\hline \multicolumn{8}{|c|}{ AWP high accuracy $(/ 1)$} \\
\hline Post-test & $0.31 \pm 0.12$ & $0.45 \pm 0.06$ & $0.41 \pm 0.10$ & & & & \\
\hline \multicolumn{8}{|c|}{ Stroop interference ratio (/1) } \\
\hline Pre-test & $0.46 \pm 0.03$ & $0.50 \pm 0.01$ & $0.52 \pm 0.02$ & 1.35 & 0.248 & 1.42 & 0.240 \\
\hline Post-test & $0.48 \pm 0.03$ & $0.49 \pm 0.01$ & $0.51 \pm 0.02$ & & & & \\
\hline \multicolumn{8}{|c|}{ Stroop interference rate $(\%)$} \\
\hline Pre-test & $3.29 \pm 1.79$ & $2.10 \pm 0.88$ & $2.34 \pm 1.38$ & 1.18 & 0.280 & $<1$ & - \\
\hline Post-test & $2.69 \pm 0.29 *$ & $2.97 \pm 0.14$ & $3.26 \pm 0.22$ & & & & \\
\hline \multicolumn{8}{|c|}{ Rule shift switch ER (\%) } \\
\hline Pre-test & $43.7 \pm 7.50$ & $19.4 \pm 3.67$ & $16.0 \pm 5.77$ & 4.25 & 0.042 & 3.35 & 0.074 \\
\hline Post-test & $27.1 \pm 8.01^{*}$ & $21.2 \pm 3.91$ & $15.6 \pm 6.17$ & & & & \\
\hline
\end{tabular}

ANOVAs' $F$ and $P$ values for the interaction between Group (CD vs. fall or CD vs. Tai) and Period (pre-vs. post-test). Asterisks indicate statistical significant difference $(N K, P<0.05)$ between pre- and post-test periods. AWP low/high: arithmetic word problems with low/high planning demand. 
when comparing CD to either fall prevention or Tai Chi Chuan $(F<1)$.

\section{Stroop test}

For the interference ratio, two-way ANOVA with Group and Period as factors did not show any statistical significant interaction for CD compared to either fall prevention $\left(F_{1,81}=1.35, P=0.248\right)$ or Tai Chi Chuan $\left(F_{1,41}=1.42, P=0.240\right)$, and post hoc tests failed to show any significant effect $(\mathrm{NK}, P>0.05$; see Table 3 ).

With respect to the interference error rate, two-way ANOVA with Group and Period as factors showed an absence of significant interaction for $\mathrm{CD}$ compared to either fall prevention
$\left(F_{1,81}=1.18, P=0.280\right)$ or Tai Chi Chuan $(F<1)$. Though the interference error rate tended to increase in the $\mathrm{CD}$ group between pre- and post-test periods, the difference did not reach statistical significance, as well as for other post hoc tests (NK, $P>0.05$; see Table 3).

\section{Rule shift cards test}

Differences in profile scores and switch error rates between preand post-test periods are shown in Figures 1A,B, respectively. Comparing CD to fall prevention, two-way ANOVA with Group (CD vs. fall) and Period as factors showed a marginally significant interaction $\left(F_{1,81}=3.82, P=0.054\right)$, and a significant difference

\section{A}

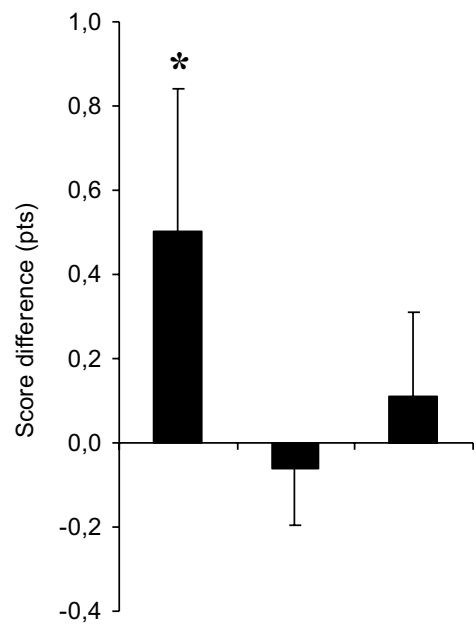

CD Fall Tai

C

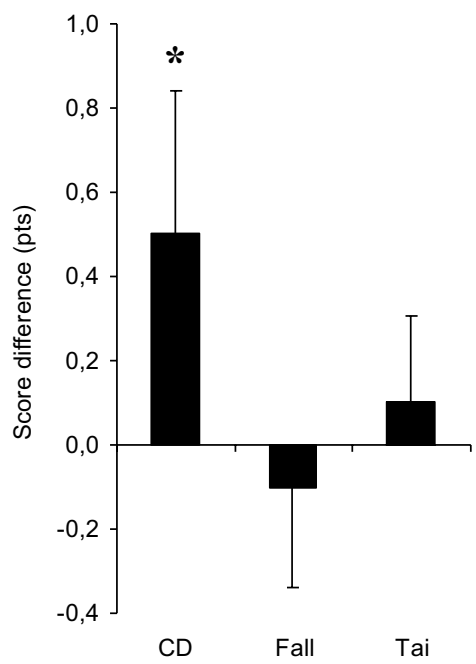

FIGURE 1 | Results for the Rule shift cards test in the main (A-B) and additional analyses (C-D). (A,C) Mean score difference in points out of 4 , and $(\mathbf{B}, \mathbf{D})$ mean switch error rate difference in points of percentage between pre-test and post-test periods for the three groups of participants: contemporary dance trainees [CD, $N=16$ in
B

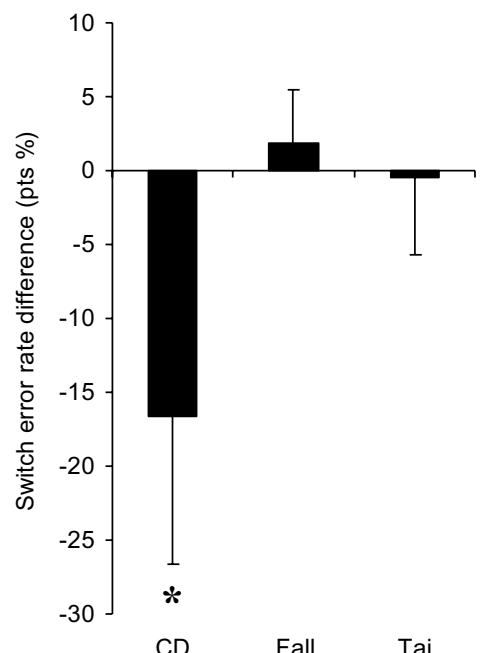

D

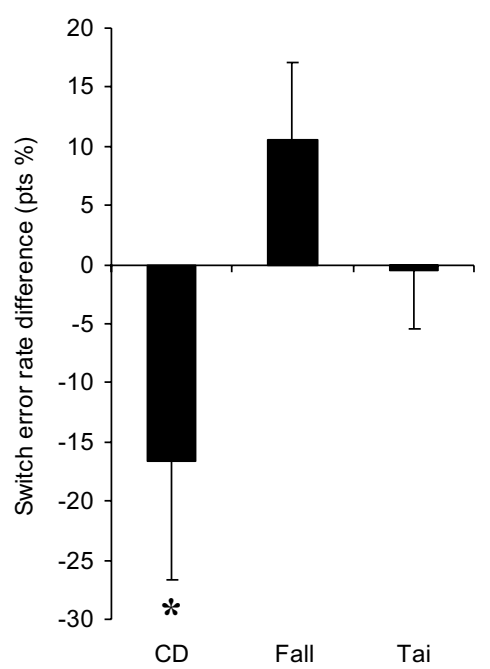

(A-D)], fall prevention trainees [Fall, $N=67$ in (A-B); $N=20$ in (C-D)], and Tai Chi Chuan trainees [Tai, $N=27$ in (A-B): $N=20$ in (C-D)].

Vertical bars are the averaged SE in the two periods. Asterisks indicate statistical significant differences between pre- and post-tests only for the $C D$ group. 
between the two periods for the CD group (NK, $P=0.016)$ but not for the fall prevention group (NK, $P=0.769)$. Importantly, scores in the pre-test period were not statistically different between the two groups (NK, $P>0.05$ ). Comparing CD to Tai Chi Chuan, two-way ANOVA with Group (CD vs. Tai) and Period as factors did not show any interaction $\left(F_{1,41}=1.46, P=0.234\right)$, but revealed a significant difference between the two periods for the CD group (NK, $P=0.034$ ), which was not the case for the Tai Chi Chuan group (NK, $P=0.628)$. Again, scores in pre-test period were statistically equivalent in the two groups $(\mathrm{NK}, P>0.05$; see Table 3).

For switch error rates, two-way ANOVA with Group (CD vs. fall) and Period as factors showed a significant interaction $\left(F_{1,81}=4.25, P=0.042\right)$, and a significant difference between the two periods for the CD group $(\mathrm{NK}, P=0.010)$ but not the fall prevention group $(\mathrm{NK}, P=0.774)$. Importantly, the switch error rates in pre-test period were not statistically different between the groups despite the tendency of the $\mathrm{CD}$ group for a higher rate (NK, $P>0.05)$. Comparing $\mathrm{CD}$ to Tai Chi Chuan, two-way ANOVA with Group (CD vs. Tai) and Period as factors showed a marginally significant interaction $\left(F_{1,41}=3.35, P=0.074\right)$, and post hoc test of the two periods was significant for the CD group (NK, $P=0.011)$ but not for Tai Chi Chuan group (NK, $P=0.948)$. Again, the rates were statistically equivalent in the two groups in pre-test period (NK, $P>0.05$; see Table 3 ).

\section{ADDITIONAL ANALYSIS}

\section{Counterbalancing effects}

One-way ANOVA with Order as factor (three levels) did not show any statistical significant difference in pre-test period on accuracy $(F<1)$ and time $\left(F_{2,46}=1.62, P>0.05\right)$ of low planning problems, on accuracy $(F<1)$ and time $\left(F_{2,16}=1.40, P>0.05\right)$ of high planning problems, on the interference ratio $(F<1)$ and the interference error rate $\left(F_{2,53}=1.08, P>0.05\right)$ of the Stroop test, and on the profile score $\left(F_{2,53}=1.01, P>0.05\right)$ and the switch error rate $\left(F_{2,53}=1.38, P>0.05\right)$ of the Rule shift cards test; as well as in the post-test on accuracy and time of low and high planning problems $(F<1)$, on the interference ratio $\left(F_{2,53}=1.50, P>0.05\right)$ and the interference error rate $(F<1)$ of the Stroop test, and on the profile score $\left(F_{2,53}=1.79, P>0.05\right)$ and the switch error rate $\left(F_{2,53}=1.00, P>0.05\right)$ of the Rule shift cards test.

\section{Arithmetic word problems}

Table 4 details the results for the three groups in the two periods. For accuracy of low planning problems, two-way ANOVAs with Group (CD vs. fall or CD vs. Tai) and Period as factors showed neither statistical significant interaction between Group and Period ( $F<1$ for both CD vs. fall and CD vs. Tai) nor significant post hoc test (NK, $P>0.05$; see Table 4). Response times were $5.20 \pm 6.97,2.92 \pm 1.39,6.12 \pm 1.25$, and $9.60 \pm 2.33,4.92 \pm 2.53$, $7.69 \pm 2.28 \mathrm{~s}$ for CD, fall prevention, and Tai Chi Chuan in pre- and post-test periods, respectively. Interaction and post hoc tests failed to reach statistical significance when comparing CD to either fall prevention or Tai Chi Chuan. For high planning problems, there was neither significant interaction nor significant post hoc tests for accuracy when comparing CD to either fall prevention or Tai Chi Chuan (see Table 4). There was not enough data to perform statistics on response times.

\section{Stroop test}

Two-way ANOVAs with Group (CD vs. fall or CD vs. Tai) and Period as factors did not show statistical significant interaction for

Table 4 | Mean \pm SE of neuropsychological tests for the groups of participants (CD: contemporary dance, $N=16$; fall: fall prevention, $N=20$; Tai: Tai Chi Chuan, $N=20$ ). Other notations as in Table 3.

\begin{tabular}{|c|c|c|c|c|c|c|c|}
\hline & \multirow[t]{2}{*}{ CD } & \multirow[t]{2}{*}{ Fall } & \multirow[t]{2}{*}{ Tai } & \multicolumn{2}{|c|}{ CD vs. fall } & \multicolumn{2}{|c|}{ CD vs. tai } \\
\hline & & & & $\boldsymbol{F}$ & $P$ & $\boldsymbol{F}$ & $P$ \\
\hline Pre-test & $0.87 \pm 0.09$ & $0.85 \pm 0.08$ & $0.90 \pm 0.08$ & $<1$ & - & $<1$ & - \\
\hline Post-test & $0.69 \pm 0.10$ & $0.70 \pm 0.10$ & $0.85 \pm 0.10$ & & & & \\
\hline AWP high & $y(/ 1)$ & & & & & & \\
\hline Post-test & $0.31 \pm 0.12$ & $0.35 \pm 0.11$ & $0.30 \pm 0.11$ & & & & \\
\hline \multicolumn{8}{|c|}{ Stroop interference ratio $(/ 1)$} \\
\hline Pre-test & $0.46 \pm 0.03$ & $0.52 \pm 0.02$ & $0.53 \pm 0.02$ & 2.77 & 0.105 & 0.180 & 0.189 \\
\hline Post-test & $0.48 \pm 0.03$ & $0.47 \pm 0.02$ & $0.51 \pm 0.02$ & & & & \\
\hline \multicolumn{8}{|c|}{ Stroop interference rate $(\%)$} \\
\hline Post-test & $2.69 \pm 0.29 *$ & $2.97 \pm 0.14$ & $3.26 \pm 0.22$ & & & & \\
\hline \multicolumn{8}{|c|}{ Rule shift switch ER (\%) } \\
\hline Pre-test & $43.7 \pm 7.50$ & $12.8 \pm 6.15$ & $12.2 \pm 6.15$ & 4.71 & 0.037 & 2.43 & 0.128 \\
\hline Post-test & $27.1 \pm 8.01^{*}$ & $23.3 \pm 7.59$ & $11.7 \pm 7.59$ & & & & \\
\hline
\end{tabular}


neither the interference ratio $\left(F_{1,34}=2.77, P=0.105\right.$ for $\mathrm{CD}$ vs. fall; $F_{1,34}=0.180, P=0.189$ for $C D$ vs. Tai) nor the interference error rate $(F<1$ for both $C D$ vs. fall and $C D$ vs. Tai). Post hoc comparisons also failed to show any significant difference (NK, $P>0.05$; see Table 4).

\section{Rule shift cards test}

Differences in profile scores and switch error rates between preand post-test periods are illustrated in Figures 1C,D, respectively. Two-way ANOVA with Group (CD vs. fall) and Period as factors showed a marginally significant interaction for the score $\left(F_{1,34}=2.88, P=0.099\right)$ and a significant interaction for the rate $\left(F_{1,34}=4.71, P=0.037\right)$. Difference between the two periods was marginally significant for the CD group (NK, $P=0.054$ for the score, and $P=0.069$ for the rate) but insignificant for the fall prevention group (NK, $P=0.692$ for the score, and $P=0.242$ for the rate). Two-way ANOVA with Group (CD vs. Tai) and Period as factors did not show any interaction for either the profile score $\left(F_{1,34}=1.21, P=0.278\right)$ or the switch error rate $\left(F_{1,34}=2.43\right.$, $P=0.128)$. Only a marginally significant or significant difference between the two periods was found for the CD group (NK, $P=0.060$ for the score, and $P=0.029$ for the rate), which was not the case for the Tai Chi Chuan group (NK, $P=0.699$ and $P=0.940$ for the score and rate, respectively). Importantly, scores and rates in pre-test period were not statistically different between the compared groups (NK, $P>0.05$; see Table 4).

\section{DISCUSSION}

The present study indicated that older adults trained in CD once a week for 5.7 months improved their switching attention or cognitive flexibility as revealed by an increase in their profile score and decrease in their switch error rate in the Rule shift cards test. This effect was task-specific as setting and suppressing attention did not benefit from CD training, and motor activity-specific as other motor training programs (fall prevention, Tai Chi Chuan) had no effect on switching attention. In the following sections, we start with a comment on methodological issues, before entering the core of the discussion describing first the impact of physical fitness vs. motor activity on attentional control, and second the hypothetical mechanisms by which motor activity, particularly CD improvisation, may influence attentional control, particularly switching attention.

The present study investigated motor training effects on subcomponents of attentional control, which we hypothesized to be a subtle phenomenon. For that reason, statistical analysis proceeded in two-by-two group comparison to search for putative training effects, comparing the experimental group (CD) with fall prevention or Tai Chi Chuan groups, separately. Such an approach is standard and used elsewhere in intervention studies comparing aquatic exercise to no activity (Hawkins et al., 1992), walking to stretching/toning (Kramer et al., 1999), folkloric dance to no activity (Eyigor et al., 2009), complex physical/perceptive/cognitive training to no activity (Marmeleira et al., 2009), or aerobic training to no activity (Renaud et al., 2010). Furthermore, our post hoc calculation used the NK method, which avoids type I errors and is more restrictive than other post hoc calculations. A second point worth discussing is the size of our samples. Intervention studies lead to much lower sample sizes than do cross-sectional ones, due to the difficulty and cost of conducting longitudinal studies together with possible dropping out during the intervention period. Our sample size was 16 in the experimental group, which was comparable to other intervention studies (e.g., $N=18$ in Hawkins et al., 1992; $N=13$ in Alpert et al., 2009; $N=19$ in Eyigor et al., 2009; $N=16$ in Marmeleira et al., 2009), though it was weaker than in other ones (e.g., $N=124 / 2$ in Kramer et al., 1999; $N=25$ in Renaud et al., 2010). It is also a general tendency that control groups have a higher sample size than the experimental group, particularly in cross-sectional studies (e.g., $N=24$ vs. 84 in Verghese, 2006; $N=24$ vs. 38 in Kattenstroth et al., 2010) for experimental and control groups, respectively). The size of our control samples were 67 and 27, thus analyses were re-run with random samples reduced to 20 in both groups to adequate with the CD group sample size. Third, our age ranges was 5989 years due to the difficulty of enrolling older adults in long-term motor training. Though studies on normal aging should ideally restrict the age range to no more than 5 or 10 years using clusters of 55-65, 66-70, 71-80 years (Colcombe and Kramer, 2003), our range (30 years) was lower or comparable to previous intervention studies (range $=36$ years in Alpert et al., 2009; 44 years in Emery et al., 2003 involving participants in cardiac rehabilitation) or cross-sectional (30 years in Roth et al., 2003; 33 years in Kattenstroth et al., 2010) studies. Some studies restricted the age range below 25 years (e.g., range $=22$ years in Marmeleira et al., 2009; 23 years in Dik et al., 2003), while others provided the average age but not the range, which might have been similar to ours given their SD (Verghese, 2006; Eyigor et al., 2009; Renaud et al., 2010). Fourth, our groups were equivalent in their past experience in motor activities. Although it cannot be ruled out that some past experience may have reduced the possibility of the our training program to impact participants' attentional control, it is noteworthy that past experience was given more detailed information in our study than in previous studies in this field of research. Finally, our motor training programs yielded equivalent social interaction. In $\mathrm{CD}$ as in fall prevention or Tai Chi Chuan, all participants were invited to work individually and in pairs, with equivalent duration between the groups in each interacting or not task.

The goal of this study was to examine the effects of motor activity on attentional control in normal aging. To achieve this goal, an experimental motor training (CD) and two control motor training programs (fall prevention and Tai Chi Chuan) were contrasted on their attentional demand. Whereas fall prevention and Tai Chi Chuan programs are constructed on codified movements and stereotyped behaviors, CD training was organized around theoretical, technical, and educational principles favoring change. Theoretically, CD training was grounded on facilitating and guiding participants in discovering their body movement potential through experience and sensations. Technically, CD training offered a series of tools (body, weight, space, time, energy, and interrelationship) whose combination determined infinite possibilities of perceiving and acting. Educationally, trainees were taught how to learn to learn how to explore their body in movement rather than asked to memorize specific skills (as in fall prevention) or movements and steps (as in Tai Chi Chuan; Ferrufino-Vargas, 2010). Importantly, emphasis was put 
on improvisation, which was twofold. The first type was the solo improvisation, practiced in steps (i) and (iv) of the improvisation stage (see Training programs section), in which movement had to obey the individual's own sensations and the internal logic of kinaesthesia. The second type was the framed improvisation practiced in steps (ii) and (iii) of the improvisation stage (see Training programs section), in which movement constantly had to adapt to the constraints (the frame) given by a delineated space and/or time on the one hand, and other participants on the other (FerrufinoVargas, 2010). Taken together, the three training programs aimed at developing motor skills through motor learning to respond better to daily life situations. Nevertheless, whereas the stereotyped behaviors of fall prevention and Tai Chi Chuan required low attentional control, CD demanded high attentional control through improvisation and creativity. To search for putative changes in attentional control before and after training, we used arithmetic word problems, the Stroop test, and the Rule shift cards test for setting, suppressing, and switching attention, respectively (Stroop, 1935; Luria and Tsvetkova, 1967; Wilson et al., 1998). Though not as specific as reaction time tests (Stuss et al., 2005), these tests have been shown to be sensitive to changes in those dimensions of attentional control in aging (Hartley and Anderson, 1983; Amieva et al., 2004; Coubard et al., 2011) and brain lesion (Allain et al., 2005; Boelen et al., 2009; Silton et al., 2010) studies. Finally, as our study focused on motor activity but not physical fitness, we did not measure cardiovascular and/or strength performance for which no change was expected during the intervention period (participants also did not participate in physical fitness during this period). Our training regimen was physically not intense and equivalent in the three training programs. In other words, the focus was put not on physical performance but on the movement per se, which leads us to the issue of physical vs. motor training effects on attention.

By investigating the influence of $\mathrm{CD}$, fall prevention, and Tai Chi Chuan on the attentional performance of older adults, the present study addresses the timely issue of how motor activity may influence attentional control. Most previous intervention studies in normal aging have investigated the effect of physical fitness on attentional control (see Introduction). In these studies, physical fitness primarily enhances aerobic capacity and cardiac output through increased stroke volume and oxygen extraction, and secondarily benefits cognitive functioning, particularly attentional control, probably by increasing the levels of neurochemicals that improve neuronal plasticity and survival (Hawkins et al., 1992; Kramer et al., 2005). Physical fitness can also be based on the development of strength, and stronger effects on attentional control have observed for combined cardiovascular and strength training (Colcombe and Kramer, 2003). Physical fitness in older adults has been shown to boost several aspects of attentional control: sharing attention after aquatic exercise (Hawkins et al., 1992), suppressing attention and conflict monitoring after walking (Colcombe et al., 2004), switching, suppressing attention and conflict monitoring after walking (Kramer et al., 1999), or preparing attention after fast walking and aerobic dancing (Renaud et al., 2010). In a meta-analysis of 18 out of 167 studies, Colcombe and Kramer (2003) concluded that attentional control was the most likely cognitive function to benefit from physical fitness, particularly in women aged 66-70 years. In contrast to physical fitness, motor activity is defined as the motor learning of skills, such as balance, motor coordination, motor flexibility, and motor speed (VoelckerRehage et al., 2010). While aerobic dancing is more prone to develop cardiovascular condition (e.g., Hagen et al., 2003; Renaud et al., 2010), other types of dance relate to motor activity as they develop balance, coordination, flexibility, or speed. Indeed, jazz dance (Alpert et al., 2009), social dance (Verghese, 2006; Kattenstroth et al., 2010), or traditional dance (Sofianidis et al., 2009) have been shown to improve balance among other functions. It can be suggested that motor activity including dance may also benefit cognition, yet the nature and extent of physical fitness vs. motor activity impact on cognition are probably different (Voelcker-Rehage et al., 2010). As outlined in the Introduction, previous studies have failed to show any effect of jazz (Alpert et al., 2009) and social dance (Verghese, 2006) on cognition. Only Kattenstroth et al. (2010) reported higher cognitive performance in social dancers compared to non-dancers. Nevertheless, this crosssectional study did not allow disentangling the effects of dancing from the predisposition of individuals to choose a dance lifestyle in early adulthood. To our knowledge, our intervention study is the first one to show that CD improvisation can improve a subcomponent of attentional control, namely switching, compared to other motor training programs. Improvisation acted as training to deal with constant change, requiring high attentional demand in both endogenous and exogenous expressions of attention, in solo and framed improvisation respectively. As a corollary of constant change, CD training may also be seen as an enriched environment (Kattenstroth et al., 2010) through the variety and richness of its content, compared to other motor training programs. Taken together, $\mathrm{CD}$ training may have developed not only the motor act itself (as in fall prevention or Tai Chi Chuan), but the way it revealed itself through its interaction with changing external conditions in solving a certain motor problem, in a way reminiscent of dexterity development (Bernstein, 1996). In this perspective, improvisation may be considered as high level dexterity, by learning the way to solve a motor problem for any situation and in any condition (Bernstein, 1996). We suggest that it was such constant adaptation that may have induced specific improvement in switching attention, contrary to fall prevention and Tai Chi Chuan based on codified movements and specific skills. By which underlying mechanisms physical fitness vs. motor activity may be related to attentional control is the issue we now discuss.

At the cell level, Black et al. (1990) appreciated differences in female rats either trained by either forced (walking on a treadmill) or voluntary exercise (free access to a running wheel) or an acrobatic program (balance beams, see-saws, rope bridges, etc.). Tissue analysis of their cerebellum indicated greater density of blood vessels (angiogenesis) after forced/voluntary exercise, whereas acrobatic learning led to greater number of synapses per cell (synaptogenesis). Further studies in animals showed that exercise training increased the levels of brain-derived neurotrophin factor and of insulin-like growth factor 1, while it decreased those of corticosteroids (Berchtold et al., 2001; Carro et al., 2001; Cotman and Berchtold, 2002). Examining cells involved in neuron survival, specifically cerebellar glia of rats, Anderson et al. (1994) showed that synaptogenesis following motor-skill learning was associated with glial hypertrophy, which was not the case of angiogenesis 
following exercise. To our knowledge, only one study in humans compared the neural correlate of physical fitness vs. motor activity in older adults (Voelcker-Rehage et al., 2010). In an fMRI cross-sectional design, the authors segregated 52 women and men aged 62-79 years in low vs. high-motor- vs. physically fit groups based on their performance in a fitness battery, and invited them to complete tasks involving attentional control and perceptual speed. High-physically fit participants showed higher activation in Brodmann' areas (BA) 10, 46, 47, and in BA 21/22, and lower activation in BA 40. In contrast, high-motor fit participants showed lower activation in BA 10,46, 47, 21/22, and in ACC, and higher activation in BA 7, 31, and 40. Taken together, these results suggested that physical fitness counteracts aging effects in prefrontal and temporal areas, while motor activity is more likely to boost visuo-motor coordination and visual-spatial integration (parietal areas) as well as motor response automatization, thus reducing the inhibitory cost supported by prefrontal areas (Voelcker-Rehage et al., 2010). These results suggest that all three training programs (CD, fall prevention, and Tai Chi Chuan) may have stimulated parietal rather than prefrontal areas, and are compatible with the absence of effects of fall prevention, Tai Chi Chuan in the three tasks of attentional control, and of CD on setting/suppressing attention. To explain the effect of CD on switching attention, we propose a prefrontal-subcortical hypothesis as follows. Motor control involves extensive areas of the central nervous system from the spinal cord to the cerebral cortex: globus pallidus, putamen, caudate nucleus, thalamus, substancia nigra, subthalamic nucleus, cerebellum, reticular formation, vestibular nuclei. At the cortical level, the supplementary motor cortex, the frontal eye fields, the DLPFC play a supramotor role in, respectively, preparing (Jenkins et al., 2000), monitoring (Schall, 2004), and controlling (Rowe et al., 2000) the movement to be produced by the primary motor cortex. For attentional control, lesion, and brain imaging studies in humans have demonstrated the critical role of prefrontal cortex in the supervisory attentional system. Interestingly, the same studies have also reported the involvement of motor areas during the performance of attentional control tasks. In a setting attention task such as the Tower of London (TOL) test, positon emission tomography (PET) studies in healthy humans showed that high planning demand was correlated to striatal regions, notably bilateral caudate nuclei (Owen et al., 1996). In another study, TOL complexity activated lateral premotor cortex in addition to DLPFC and rostral ACC (Dagher et al., 1999). Using the Stroop test, Ravnkilde et al. (2002) have shown in a PET study in healthy humans the activation of left ACC, supplementary motor cortex, thalamus, and cerebellum. The involvement of subcortical motor areas was

\section{REFERENCES}

Alexander, G. E., DeLong, M. R., and Strick, P. L. (1986). Parallel organization of functionally segregated circuits linking basal ganglia and cortex. Annu. Rev. Neurosci. 9, 357-381.

Allain, P., Verny, C., Aubin, G., Pinon, K., Bonneau, D., Dubas, F., and Le Gall, D. (2005). Arithmetic wordproblem-solving in Huntington's disease. Brain Cogn. 57, 1-3.
Alpert, P. T., Miller, S. K., Wallmann, H., Havey, R., Cross, C., Chevalia, T., Gillis, C. B., and Kodandapari, K. (2009). The effect of modified jazz dance on balance, cognition, and mood in older adults. J. Am. Acad. Nurse Pract. 21, 108-115.

Amieva, H., Phillips, L. H., Della Sala, S., and Henry, J. D. (2004). Inhibitory functioning in Alzheimer's disease. Brain 127, 949-964.

corroborated by another PET study in healthy humans showing that the highest correlations could be found between time spent performing the Stroop interference task and net blood-brain clearance of fluoro-DOPA of striatal areas, namely caudate nucleus and putamen (Vernaleken et al., 2007). Finally using a switching attention task, part B of the Trail making test, the same study reported higher activation in the caudate nucleus and putamen (Vernaleken et al., 2007), while a fMRI study showed the involvement of left DLPFC and of medial frontal cortex (Zakzanis et al., 2005). Taken together, these studies using different tasks provide neurobiological evidence for an involvement of not only prefrontal cortex but also subcortical motor areas in attentional control. In addition to parietal areas and compatible with higher automated motor responses (Voelcker-Rehage et al., 2010), we suggest that motor activity may also stimulate prefrontal-subcortical loops, specifically the dorsolateral prefrontal loop linking DLPFC to striatum, pallidum, substancia nigra, and thalamus (Alexander et al., 1986). In $\mathrm{CD}$ training requiring higher attentional demand than other motor training programs, the prefrontal-subcortical interaction may have been reinforced with higher frequency and intensity, resulting in enhanced switching attention. Thus, it is the higher attentional demand, flexibility, and creativity exercised in improvisation which may have differentiated between CD and other motor training in their impact on attention. Based on animal studies, we suggest that such over-recruitment of the prefrontal-subcortical loop in CD improvisation may have acted through synaptogenesis and glial hypertrophia (Black et al., 1990; Anderson et al., 1994).

To conclude, this study was the first intervention study in normal aging to examine the influence of dance on attentional control, as compared to other motor training programs. Our results suggest that high attentional dance improvisation improves switching attention in older adults, whereas motor-skill learning based on stereotyped behaviors does not. CD improvisation may be a useful way to boost cognitive flexibility known to decline even in high-functioning older adults (Coubard et al., 2011) with helpful impact in daily life (Vaughan and Giovanello, 2010). How our result can generalize pathological conditions such as Alzheimer's disease is a challenging issue for future studies.

\section{ACKNOWLEDGMENTS}

Results were presented at the 6th International Conference on the Arts in Society (Berlin, Germany, 2011) and at the Urumqi Clinical Medicine Symposium (Urumqi, Xinjiang, China, 2011). The authors thank ADAL and L. Nosjean (Paris, France) for allowing the assessment of some participants.

Anderson, B. J., Li, X., Alcantara, A. A., Isaacs, K. R., Black, J. E., and Greenough, W. T. (1994). Glial hypertrophy is associated with synaptogenesis following motor-skill learning, but not with angiogenesis following exercise. Glia 11, 73-80.

Ashendorf, L., Jefferson, A. L., O'Connor, M. K., Chaisson, C., Green, R. C., and Stern, R. A. (2008). Trail making test errors in normal aging, mild cognitive impairment, and dementia. Arch. Clin. Neuropsychol. 23, 129-137.

Aubin, G., Le Gall, D., and Guyard, H. (1994). Etude de la résolution de problèmes numériques chez des patients frontaux. Rev. Neuropsychol. 4, 437-467.

Berchtold, N. C., Kesslak, J. P., Pike, C. J., Adlard, P. A., and Cotman, C. W. (2001). Estrogen and exercise 
interact to regulate brain-derived neurotrophic factor mRNA and protein expression in the hippocampus. Eur. J. Neurosci. 14, 1992-2002.

Bernstein, N. A. (1996). "Dexterity and its features," in Dexterity and its Development, eds M. L. Latash, M. T. Turvey, and N. A. Bernstein (Mahwah: Lawrence Erlbaum), 207-235.

Bherer, L., and Belleville, S. (2004). Age-related differences in response preparation: the role of time uncertainty. J. Gerontol. B. Psychol. Sci. Soc. Sci. 59, P66-P74.

Bherer, L., Belleville, S., and Hudon, C. (2004). Le déclin des fonctions exécutives au cours du vieillissement normal, dans la maladie d'Alzheimer et dans la démence frontotemporale. Psychol. Neuropsychiatr. Vieil. 2, 181-189.

Bherer, L., Kramer, A. F., Peterson, M. S., Colcombe, S., Erickson, K., and Becic, E. (2005). Training effects on dual-task performance: are there age-related differences in plasticity of attentional control? Psychol. Aging 20, 695-709.

Bherer, L., Kramer, A. F., Peterson, M. S., Colcombe, S., Erickson, K., and Becic, E. (2006). Testing the limits of cognitive plasticity in older adults: application to attentional control. Acta Psychol. (Amst.) 123, 261-278.

Bherer, L., Kramer, A. F., Peterson, M. S., Colcombe, S., Erickson, K., and Becic, E. (2008). Transfer effects in task-set cost and dual-task cost after dual-task training in older and younger adults: further evidence for cognitive plasticity in attentional control in late adulthood. Exp. Aging Res. 34, 188-219.

Black, J. E., Isaacs, K. R., Anderson, B. J., Alcantara, A. A., and Greenough, W. T. (1990). Learning causes synaptogenesis, whereas motor activity causes angiogenesis, in cerebellar cortex of adult rats. Proc. Natl. Acad. Sci. U.S.A. 87, 5568-5572.

Blake, R., Rizzo, M., and McEvoy, S. (2008). Aging and perception of visual form from temporal structure. Psychol. Aging 23, 181-189.

Boelen, D. H., Spikman, J. M., Rietveld, A. C., and Fasotti, L. (2009). Executive dysfunction in chronic braininjured patients: assessment in outpatient rehabilitation. Neuropsychol. Rehabil. 19, 625-644.

Bugg, J. M., Zook, N. A., DeLosh, E. L., Davalos, D. B., and Davis, H. P. (2006). Age differences in fluid intelligence: contributions of general slowing and frontal decline. Brain Cogn. 62, 9-16.

Carro, E., Trejo, J. L., Busiguina, S., and Torres-Aleman, I. (2001). Circulating insulin-like growth factor
I mediates the protective effects of physical exercise against brain insults of different etiology and anatomy. J. Neurosci. 21, 5678-5684.

Chaparro, A., Wood, J. M., and Carberry, T. (2005). Effects of age and auditory and visual dual tasks on closedroad driving performance. Optom. Vis. Sci. 82, 747-754.

Churchill, J. D., Galvez, R., Colcombe, S., Swain, R. A., Kramer, A. F., and Greenough, W. T. (2002). Exercise, experience and the aging brain. $\mathrm{Neu}$ robiol. Aging 23, 941-955.

Colcombe, S., and Kramer, A. F. (2003). Fitness effects on the cognitive function of older adults: a meta-analytic study. Psychol. Sci. 14, 125-130.

Colcombe, S. J., Kramer, A. F., Erickson, K. I., Scalf, P., McAuley, E., Cohen, N. J., Webb, A., Jerome, G. J., Marquez, D. X., and Elavsky, S. (2004). Cardiovascular fitness, cortical plasticity, and aging. Proc. Natl. Acad. Sci. U.S.A. 101, 3316-3321.

Cotman, C. W., and Berchtold, N. C. (2002). Exercise: a behavioral intervention to enhance brain health and plasticity. Trends Neurosci. 25, 295-301.

Coubard, O. A., Ferrufino, L., Boura, M., Gripon, A., Renaud, M., and Bherer, L. (2011). Attentional control in normal aging and Alzheimer's disease. Neuropsychology 25, 353-367.

Dagher, A., Owen, A. M., Boecker, H., and Brooks, D. J. (1999). Mapping the network for planning: a correlational PET activation study with the Tower of London task. Brain 122, 1973-1987.

Dik, M., Deeg, D. J., Visser, M., and Jonker, C. (2003). Early life physical activity and cognition at old age. J. Clin. Exp. Neuropsychol. 25, 643-653.

Emery, C. F., Hsiao, E. T., Hill, S. M., and Frid, D. J. (2003). Shortterm effects of exercise and music on cognitive performance among participants in a cardiac rehabilitation program. Heart Lung 32, 368-373.

Erickson, K. I., Colcombe, S. J., Wadhwa, R., Bherer, L., Peterson, M. S., Scalf, P. E., Kim, J. S., Alvarado, M., and Kramer, A. F. (2007). Training-induced functional activation changes in dual-task processing: an FMRI study. Cereb. Cortex 17, 192-204.

Eyigor, S., Karapolat, H., Durmaz, B., Ibisoglu, U., and Cakir, S. (2009). A randomized controlled trial of Turkish folklore dance on the physical performance, balance, depression and quality of life in older women. Arch. Gerontol. Geriatr. 48, 84-88.
Ferrufino-Vargas, L. (2010). "De los fundamentos de la danza contemporánea a la improvisación," in $L a$ mujer en las artes visuales y escénicas: transgresión, pluralidad y compromiso social, ed. O. Barrios (Madrid: Editorial Fundamentos), 271-291.

Folstein, M. F., Folstein, S. E., and McHugh, P. R. (1975). "Mini-mental state.” A practical method for grading the cognitive state of patients for the clinician. J. Psychiatr. Res. 12, 189-198.

Hagen, B., Armstrong-Esther, C., and Sandilands, M. (2003). On a happier note: validation of musical exercise for older persons in long-term care settings. Int. J. Nurs. Stud. 40 , 347-357.

Hank, K. (2011). How "successful" do older Europeans age? Findings from SHARE. J. Gerontol. B Psychol. Sci. Soc. Sci. 66, 230-236.

Hartley, A. A., and Anderson, J. W. (1983). Task complexity and problem-solving performance in younger and older adults. J. Gerontol. $38,72-77$.

Hawkins, H. L., Kramer, A. F., and Capaldi, D. (1992). Aging, exercise, and attention. Psychol. Aging 7, 643-653.

Hillman, C. H., Erickson, K. I., and Kramer, A. F. (2008). Be smart, exercise your heart: exercise effects on brain and cognition. Nat. Rev. Neurosci. 9, 58-65.

Houston, S. (2005). Dance for older people. Prim. Health Care 15, 18-19.

Hsiao-Wecksler, E. T. (2008). Biomechanical and age-related differences in balance recovery using the tether-release method. J. Electromyogr. Kinesiol. 18, 179-187.

Jenkins, I. H., Jahanshahi, M., Jueptner, M., Passingham, R. E., and Brooks, D. J. (2000). Self-initiated versus externally triggered movements. II. The effect of movement predictability on regional cerebral blood flow. Brain 123, 1216-1228.

Kalafat, M., Hugonot-Diener, L., and Poitrenaud, J. (2003). Standardisation et étalonnage français du "Mini Mental State" (MMS) version GRECO. Rev. Neuropsychol. 13, 209-236.

Kattenstroth, J. C., Kalisch, T., Tegenthoff, M., and Dinse, H. R. (2009). Dance therapy for cognitive enhancement in the elderly. Front. Neurosci. 3, 100-101.

Kattenstroth, J. C., Kolankowska, I., Kalisch, T., and Dinse, H. R. (2010). Superior sensory, motor, and cognitive performance in elderly individuals with multi-year dancing activities. Front. Aging Neurosci. 2:31. doi: 10.3389/fnagi.2010.00031
Kramer, A. F., Colcombe, S. J., McAuley, E., Scalf, P. E., and Erickson, K. I. (2005). Fitness, aging and neurocognitive function. Neurobiol. Aging 26(Suppl. 1), 124-127.

Kramer, A. F., and Erickson, K. I. (2007). Capitalizing on cortical plasticity: influence of physical activity on cognition and brain function. Trends Cogn. Sci. 11, 342-348.

Kramer, A. F., Erickson, K. I., and Colcombe, S. J. (2006). Exercise, cognition, and the aging brain. J. Appl. Physiol. 101, 1237-1242.

Kramer, A. F., Hahn, S., Cohen, N. J., Banich, M. T., McAuley, E., Harrison, C. R., Chason, J., Vakil, E., Bardell, L., Boileau, R. A., and Colcombe, A. (1999). Ageing, fitness and neurocognitive function. Nature 400, 418-419.

Lezak, M. D. (1995). Neuropsychological Assessment. New York: Oxford University Press.

Libster, M. (2006). Dancing away dispiritedness: dance, both social and therapeutic improves more than physical and mental function in the elderly. Am. J. Nurs. 106, 72C-D.

Lima, M., and Vieira, A. (2007). Ballroom dance as therapy for the elderly in Brazil. Am. J. Dance Ther. 29, 129-142.

Lister, J. P., and Barnes, C. A. (2009). Neurobiological changes in the hippocampus during normative aging. Arch. Neurol. 66, 829-833.

Luria, A. R., and Tsvetkova, L. S. (1967). Les troubles de la résolution de problèmes. Analyse neuropsychologique. Paris: Gauthier-Villars.

Maquestiaux, F., Lague-Beauvais, M., Ruthruff, E., and Bherer, L. (2008). Bypassing the central bottleneck after single-task practice in the psychological refractory period paradigm: evidence for task automatization and greedy resource recruitment. Mem. Cognit. 36, 1262-1282.

Maquestiaux, F., Lague-Beauvais, M., Ruthruff, E., Hartley, A., and Bherer, L. (2010). Learning to bypass the central bottleneck: declining automaticity with advancing age. Psychol. Aging 25, 177-192.

Marmeleira, J. F., Godinho, M. B., and Fernandes, O. M. (2009). The effects of an exercise program on several abilities associated with driving performance in older adults. Accid. Anal. Prev. 41, 90-97.

Meulemans, T. (2008). "La batterie GREFEX: présentation générale," in Fonctions Exécutives et Pathologies Neurologiques et Psychiatriques. Evaluation en Pratique Clinique, ed. O. Godefroy and GREFEX (Marseille: Solal), 217-229. 
Mozolic, J. L., Hayasaka, S., and Laurienti, P. J. (2010). A cognitive training intervention increases resting cerebral blood flow in healthy older adults. Front. Hum. Neurosci. 4:16. doi: 10.3389/neuro.09.016.2010

Neeper, S. A., Gomez-Pinilla, F., Choi, J., and Cotman, C. (1995). Exercise and brain neurotrophins. Nature 373, 109.

Oken, B. S., Zajdel, D., Kishiyama, S., Flegal, K., Dehen, C., Haas, M., Kraemer, D. F., Lawrence, J., and Leyva, J. (2006). Randomized, controlled, six-month trial of yoga in healthy seniors: effects on cognition and quality of life. Altern. Ther. Health Med. 12, 40-47.

Owen, A. M., Doyon, J., Petrides, M., and Evans, A. C. (1996). Planning and spatial working memory: a positron emission tomography study in humans. Eur. J. Neurosci. 8, 353-364.

Parasuraman, R. (2000). The Attentive Brain. Cambridge, MA: MIT Press.

Rajah, M. N., and D'Esposito, M. (2005). Region-specific changes in prefrontal function with age: a review of PET and fMRI studies on working and episodic memory. Brain 128, 1964-1983.

Ravnkilde, B., Videbech, P., Rosenberg, R., Gjedde, A., and Gade, A. (2002). Putative tests of frontal lobe function: a PET-study of brain activation during Stroop's test and verbal fluency. J. Clin. Exp. Neuropsychol. 24, 534-547.

Raz, N., and Rodrigue, K. M. (2006). Differential aging of the brain: patterns, cognitive correlates and modifiers. Neurosci. Biobehav. Rev. 30, 730-748.

Renaud, M., Maquestiaux, F., Joncas, S., Kergoat, M. J., and Bherer, L. (2010). The effect of three months of aerobic training on response preparation in older adults. Front. Aging Neurosci. 2:148. doi: 10.3389/fnagi.2010.00148

Roth, D. L., Goode, K. T., Clay, O. J., and Ball, K. K. (2003). Association of physical activity and visual attention in older adults. J. Aging Health 15, 534-547.

Rowe, J. B., Toni, I., Josephs, O., Frackowiak, R. S., and Passingham, R. E. (2000). The prefrontal cortex: response selection or maintenance within working memory? Science 288, 1656-1660.

Rypma, B., Eldreth, D. A., and Rebbechi, D. (2007). Age-related differences in activation-performance relations in delayed-response tasks: a multiple component analysis. Cortex 43, 65-76.

Schäfer, S., Huxhold, O., and Lindenberger, U. (2006). Healthy mind in healthy body? A review of sensorimotor-cognitive interdependencies in old age. Eur. Rev. Aging Phys. Act. 3, 45-54.

Schall, J. D. (2004). On the role of frontal eye field in guiding attention and saccades. Vision Res. 44, 1453-1467.

Silton, R. L., Heller, W., Towers, D. N., Engels, A. S., Spielberg, J. M., Edgar, J. C., Sass, S. M., Stewart, J. L., Sutton, B. P., Banich, M. T., and Miller, G. A. (2010). The time course of activity in dorsolateral prefrontal cortex and anterior cingulate cortex during top-down attentional control. Neuroimage 50, 1292-1302.

Sofianidis, G., Hatzitaki, V., Douka, S., and Grouios, G. (2009). Effect of a 10-week traditional dance program on static and dynamic balance control in elderly adults. J. Aging Phys. Act. 17, 167-180.

Sorel, O., and Pennequin, V. (2008). Aging of the planning process: the role of executive functioning. Brain Cogn. 66, 196-201.

Spirduso, W. W. (1975). Reaction and movement time as a function of age and physical activity level. J. Gerontol. 30, 435-440.

Stacey, G., and Stickley, T. (2008). Dancing to keep young at heart. Ment. Health Pract. 11, 34-38.
Stroop, J. R. (1935). Studies of interference in serial verbal reactions. J. Exp. Psychol. 18, 643-662.

Stuss, D. T., and Alexander, M. P. (2007). Is there a dysexecutive syndrome? Philos. Trans. R. Soc. Lond. B Biol. Sci. 362, 901-915.

Stuss, D. T., Alexander, M. P., Shallice, T., Picton, T. W., Binns, M. A., Macdonald, R., Borowiec, A., and Katz, D. I. (2005). Multiple frontal systems controlling response speed. Neuropsychologia 43, 396-417.

Stuss, D. T., Shallice, T., Alexander, M. P., and Picton, T. W. (1995). A multidisciplinary approach to anterior attentional functions. Ann. N. Y. Acad. Sci. 769, 191-211.

Tentori, K., Osherson, D., Hasher, L. and May, C. (2001). Wisdom and aging: irrational preferences in college students but not older adults. Cognition 81, B87-B96.

Vallesi, A., McIntosh, A. R., and Stuss, D. T. (2009). Temporal preparation in aging: a functional MRI study. Neuropsychologia 47, 2876-2881.

Vaughan, L., and Giovanello, K. (2010). Executive function in daily life: age-related influences of executive processes on instrumental activities of daily living. Psychol. Aging 25, 343-355.

Verghese, J. (2006). Cognitive and mobility profile of older social dancers. J. Am. Geriatr. Soc. 54, 1241-1244.

Verhaeghen, P., and Cerella, J. (2002). Aging, executive control, and attention: a review of meta-analyses. $\mathrm{Neu}$ rosci. Biobehav. Rev. 26, 849-857.

Vernaleken, I., Buchholz, H. G., Kumakura, Y., Siessmeier, T., Stoeter, P., Bartenstein, P., Cumming, P., and Grunder, G. (2007). 'Prefrontal' cognitive performance of healthy subjects positively correlates with cerebral FDOPA influx: an exploratory [18F]-fluoro-L-DOPAPET investigation. Hum. Brain Mapp. 28, 931-939.

Voelcker-Rehage, C., Godde, B., and Staudinger, U. M. (2010). Physical and motor fitness are both related to cognition in old age. Eur. J. Neurosci. 31, 167-176.

Wechsler, D. (1989). Manuel de l'échelle d'intelligence de Wechsler pour adultes - forme révisée (WAIS$R)$. Paris: Editions du Centre de Psychologie Appliquée.

Wilson, B. A., Evans, J. J., Emslie, H., Alderman, N., and Burgess, P. (1998). The development of an ecologically valid test for assessing patients with dysexecutive syndrome. Neuropsychol. Rehabil. 8, 213-228.

Zakzanis, K. K., Mraz, R., and Graham, S. J. (2005). An fMRI study of the trail making test. Neuropsychologia 43, 1878-1886.

Zook, N., Welsh, M. C., and Ewing, V. (2006). Performance of healthy, older adults on the Tower of London Revised: associations with verbal and nonverbal abilities. Neuropsychol. Dev. Cogn. B Aging Neuropsychol. Cogn. 13, $1-19$.

Conflict of Interest Statement: The authors declare that the research was conducted in the absence of any commercial or financial relationships that could be construed as a potential conflict of interest.

Received: 21 March 2011; accepted: 01 September 2011; published online: 20 September 2011.

Citation: Coubard OA, Duretz S, Lefebvre $V$, Lapalus $P$ and Ferrufino $L$ (2011) Practice of contemporary dance improves cognitive flexibility in aging. Front. Ag. Neurosci. 3:13. doi: 10.3389/fnagi.2011.00013

Copyright (c) 2011 Coubard, Duretz, Lefebvre, Lapalus and Ferrufino. This is an open-access article subject to a nonexclusive license between the authors and Frontiers Media SA, which permits use, distribution and reproduction in other forums, provided the original authors and source are credited and other Frontiers conditions are complied with. 